\title{
WEB-BASED COMPUTER VISUAL SIMULATOR
} An Education Tool with Java Technologies

\author{
Yoshiro Imai, Shinji Tomita, Haruo Niimi and Toshiaki Kitamura \\ Imai:imai@eng.kagawa-u.ac.jp, Kagawa University, 2216-20 Hayashi-cho Takamatsu 761- \\ 0396 Japan; Tomita: Kyoto University, GraduateSchool of Informatics, Yoshida honmachi \\ Sakyo-ku Kyoto 606-8501 Japan Niimi: Kyoto Sangyou University, Kamigamo Motoyama \\ Kita-ku Kyoto 603-8555 Japan; Kitamura: Hiroshima City University, Ohtsuka Higashi \\ 3chome Asaminami-ku Hiroshima731-3194 Japan
}

\begin{abstract}
A visual simulator has been developed as an example of Web-based education tool, which is designed for students to understand graphically internal behavior and structure of computer system in a course of information engineering education. It is written in Java programming language, downloadable from Web server and executable on almost all types of personal computers including Java virtual machine and major Web browsers.
\end{abstract}

Key words: Web-based Education Tool, Visual Simulator, Java Applet, Built-in Mail Facility for Communication between Users, Trial Application to Collaborative Education

\section{INTRODUCTION}

Recently, actual computers become higher-performance and more complicated. It is not easy for students to understand how computer works because of its complex black-box-like structure. An abstract lecture on computer cannot provide visual and applicable understanding on computer and assembly programming. In order to study computer more precisely, it is necessary to illustrate the internal behavior and structure of computer.

We have developed a visual simulator called "VisuSim" as Web-based education tool for computer system. It is implemented in Java programming language. With this tool, it is useful for students to understand visually the internal behavior and structure of computer. VisuSim provides window- 
oriented graphical view to explain how computer works, for example, to interpret sample assembly programs and to illustrate data transfer mechanism between registers of CPU and memory. It has been designed to fulfill its function not only as stand-alone application on several platforms but also as Java applet on major browsers.

This paper describes the basic design concepts and existing facilities of our proposed visual simulator. It illustrates characteristics of its GUI for actual manipulation, explains simulating facilities with two kinds of execution modes, and demonstrates details of newly enhanced facilities such as on-line help guide and embedded mail handler. Finally, the application of VisuSim to practical lectures and current evaluation as Web-based education tool for computer system and/or assembly programming are also shown.

\section{DESIGN OF WEB-BASED EDUCATION TOOL}

First of all, design concepts of our simulator "VisuSim" are summarized as follows [1]:

a) It is used as a practical education tool, which ranges from lectures of computer system to exercises of assembly programming. It illustrates internal behavior and structure of von Neumann-style computer;

b) It is equipped with a simple GUI for interactive manipulations which includes initialization, program loading, and execution of program. It simulates stepwise and/or continuous execution of programs and provides an effective environment for assembly program debugging;

c) It is implemented by means of a single source code in Java language which can be used not only as stand-alone application but as Java applet. It is easily distributed from Web server into users through network connectivity;

d) It is independent from the type of operating system and/or machine specification and is also available in multiple platform environments. It works on major Web browsers such as Microsoft IE and Netscape Composer/Navigator so that it does not need additional software.

Although many of these concepts are not original ideas for our simulator, they are efficient ideas for designing education tools and effective methods for utilizing tools in the practical situation. We know that simulation-based learning is one of the most efficient ways to educate student from university to elemental school [1][2]. There is the same idea to develop a visual simulator with Java for understanding behavior and structure of internal computer [3]. But it is our original approach to design visual simulator in order to work as both stand-alone and Java applet in order to be used in the 
classroom lecture, after school, or at home. For example, VisuSim may be invoked as stand-alone application. In this case, it can read local files of sample programs and/or help message. In another case, VisuSim may be downloaded from Web server and be running as Java applet. It can obtain sample programs and/or help messages from its Web server and execute and/or displaying them on the browser. Figure 1 shows a scheme of both ways to utilize VisuSim.

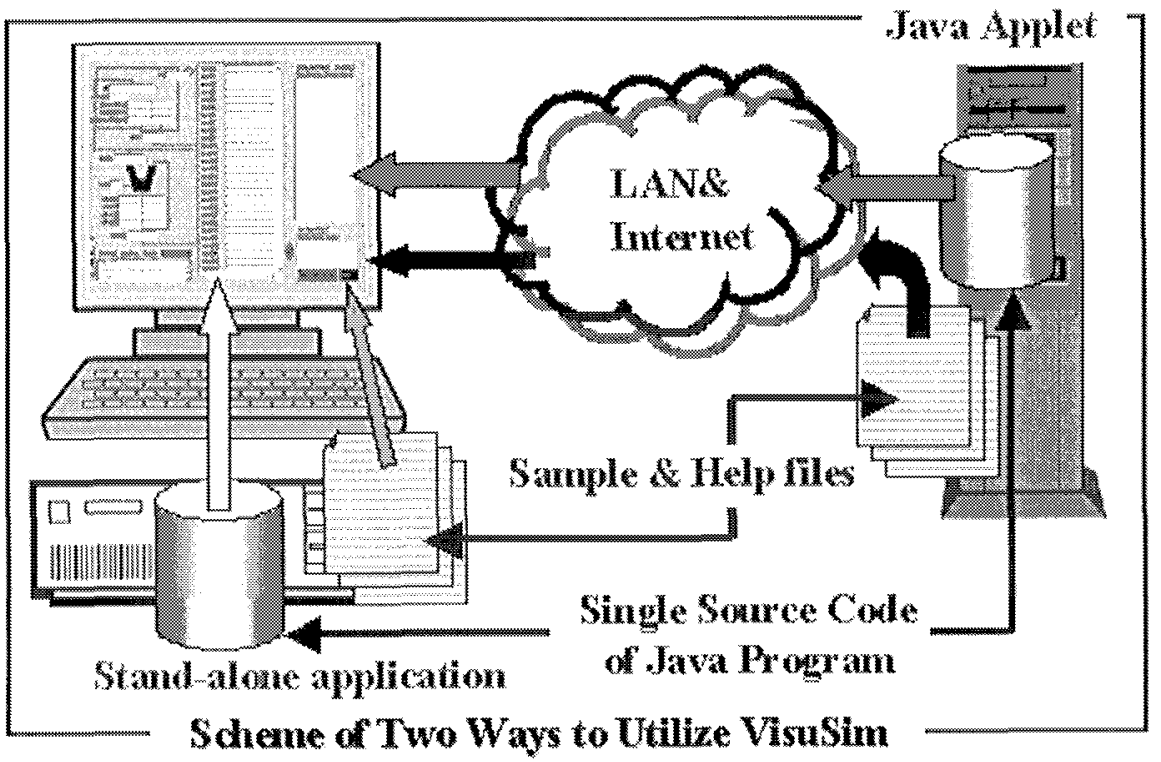

Figure1. Scheme of Both ways to utilize Education Tool: VisuSim

Secondly, the existing features of the simulator are illustrated on a practical example. With our visual simulator, it is graphically demonstrated how computer works, namely; loading program into memory; fetching each instruction of program in the memory; decoding it into several fields such as operation code and address fields; and executing accordingly to the result of decoding instructions.

Its on-line help mechanism is implemented to allow VisuSim to read a specific file and display its contents on the newly created window. Teachers can rewrite such a file in order to customize the help message of VisuSim for each student, and select suitable files of sample codes according to student's understanding level. 


\section{CAPABILITY OF THE SIMULATOR VISUSIM}

\subsection{GUI of VisuSim and its simulation modes}

A GUI of VisuSim plays two roles as follows; a) to provide information about simulated computer and b) to control the VisuSim. Figure 2 shows an applet-based GUI of VisuSim.

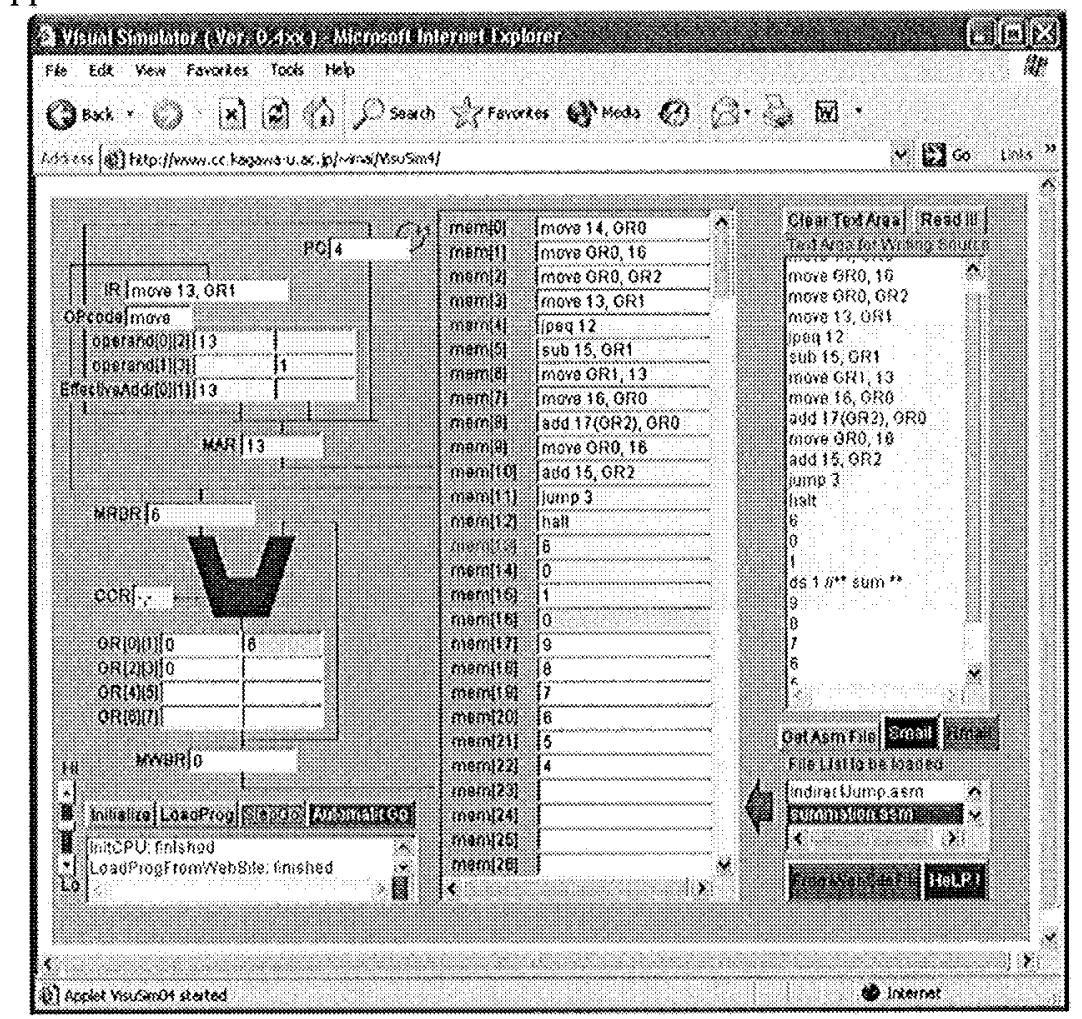

Figure2. GUI of VisuSim running on Applet

The former feature is constructed with several labels and text fields which configures corresponding registers in internal structure of computer. Control Unit including program counter (PC) and instruction register (IR) is allocated at the upper right hand of GUI. Processor including ALU and registers (GRs) is located below Control Unit. The middle position of GUI is for main memory, which is laid on sliding panel.

The latter feature of GUI consists of buttons and other objects for information interchanging between the simulator and user. Major four buttons are placed on the lower right hand of GUI, which are assigned for 
initializing CPU, loading program to memory, executing program step-bystep, and executing autonomously, respectively.

It is indispensable to provide such a sufficient way that students can correctly use GUI including buttons, fields and so on. Display of guideline for users is effective and important in order to explain the purpose of each feature in the GUI and to indicate how to use it.

\subsection{Two kinds of Simulation modes}

VisuSim provides two different kinds of simulation modes: a step-bystep execution of program (called "stepwise mode") and a continuous execution (called "automatic mode"). The former is convenient for tracing the program step-by-step and finding illegal points of program, while the latter is helpful to execute a series of code blocks continuously in order to confirm whether a whole program works correctly or not.

Two kinds of simulation modes are summarized as follows;

(1) Stepwise mode execution:

When the button for "Step Go" is clicked, the internal simulator module of VisuSim activates only one machine cycle execution. A machine cycle of this mode strictly consists of instruction fetching, instruction decoding and execution. Sequential execution of the specific part of program needs consecutive operations of clicking the button for "Step Go". This simulation mode is suitable for interactive modification of registers and/or memory while breaking the execution. Namely, it is convenient to stop the execution of a program at any point, change contents of memory and/or CPU (including several registers), and then restart from such the previous break point. This mode could make VisuSim compute only the limited part in the long size code of program, if program counter is set to the specific location and the button for "Step Go" is being clicked during the suitable times. Such a process can point out the illegal description of programs, offer a better modification for improving, and finally prove code consistency of those examined programs. A detail testing of program can be realized in this mode. For example, it is useful to understand how a computer works.

(2) Automatic mode execution:

When the button for "Automatic Go" is clicked, the internal simulator of VisuSim starts continuous repetition of machine cycle execution until decoding halt instruction or occurrence of interruption. This mode is much useful for a relatively long-term demonstration where VisuSim must interpret a program including, for example, several iterations, recursive procedure calls or other kind of complicated processes. Because this mode of execution looks like playing slide show, it is helpful to trace a whole target program sequentially and verify whether the program is computed accurately 
or not. While VisuSim repeats its machine cycles in the automatic execution mode, pushing the button for "Automatic Go" can trigger occurrence of interruption. At the interruption of VisuSim, pushing again such a button allows VisuSim to restart its machine cycles from the previous interrupted point. Speed control is available on a slide bar and can be varied from higher level to lower one. Each personal computer has various CPU with different type of frequency. It is useful to adjust the simulation speed of VisuSim in accordance with the different execution environments.

\subsection{Communication with VisuSim's Mailing Facility}

The mail handling facility embedded in VisuSim has been realized with pure Java code and consists of two major parts below, namely, SMTP-based mail sender module and POP3-based mail receiver one.

(1) Characteristics of SMTP-based mail sender:

This mail sender is different from other general-purpose mailers' sending modules at the following two points. At first, when students, who use VisuSim, meet some problems and want to consult teacher or friends about such a situation, they can simply transfer the current state of simulator in VisuSim (including data of all the registers and memory) into a mail to be sent. And moreover, by means of sending e-mail with the status of VisuSim, reports of students are easily submitted to teacher in the lecture of computer system and/or assembly programming exercise. Data in the content of the received mail can be analyzed in order to investigate whether student understands or not by teacher who received such a mail. This is a major reason to develop a special-purpose embedded mailer for VisuSim.

Secondly, when users want to write e-mail with SMTP-based mail sender of VisuSim, they may specify the e-mail address not only as domain-name oriented notation such as "imai@eng.kagawa-u.ac.jp" but also as IP address specific notation such as "imai@)(192.168.0.3". It is very useful for users to send e-mail with SMTP-based sender provided by VisuSim, because almost all temporary (informal) mail servers for users of VisuSim are not always entered on the official Domain Name Server and therefore such a mail server must be specified with IP address only.

As you know, there is still a problem for SMTP-based sender invoked from VisuSim which is running on Java applet. Such an applet is so tightly limited on network activity that it cannot access other servers except of the Web server which actually distributes VisuSim's applet code to user's browser. In order to improve such a case and realize communication by email, the Web server will be also required to be equipped together with mail service facility in our system. In the case of VisuSim running as stand-alone application, however, users can be released from this restricted usage of 
VisuSim. It is available for users of VisuSim to send their e-mail to any server they want by means of SMTP-based sender.

Figure 3 illustrates a scheme that SMTP-based mail sender performs sending e-mail, which is invoked from VisuSim running on applet.

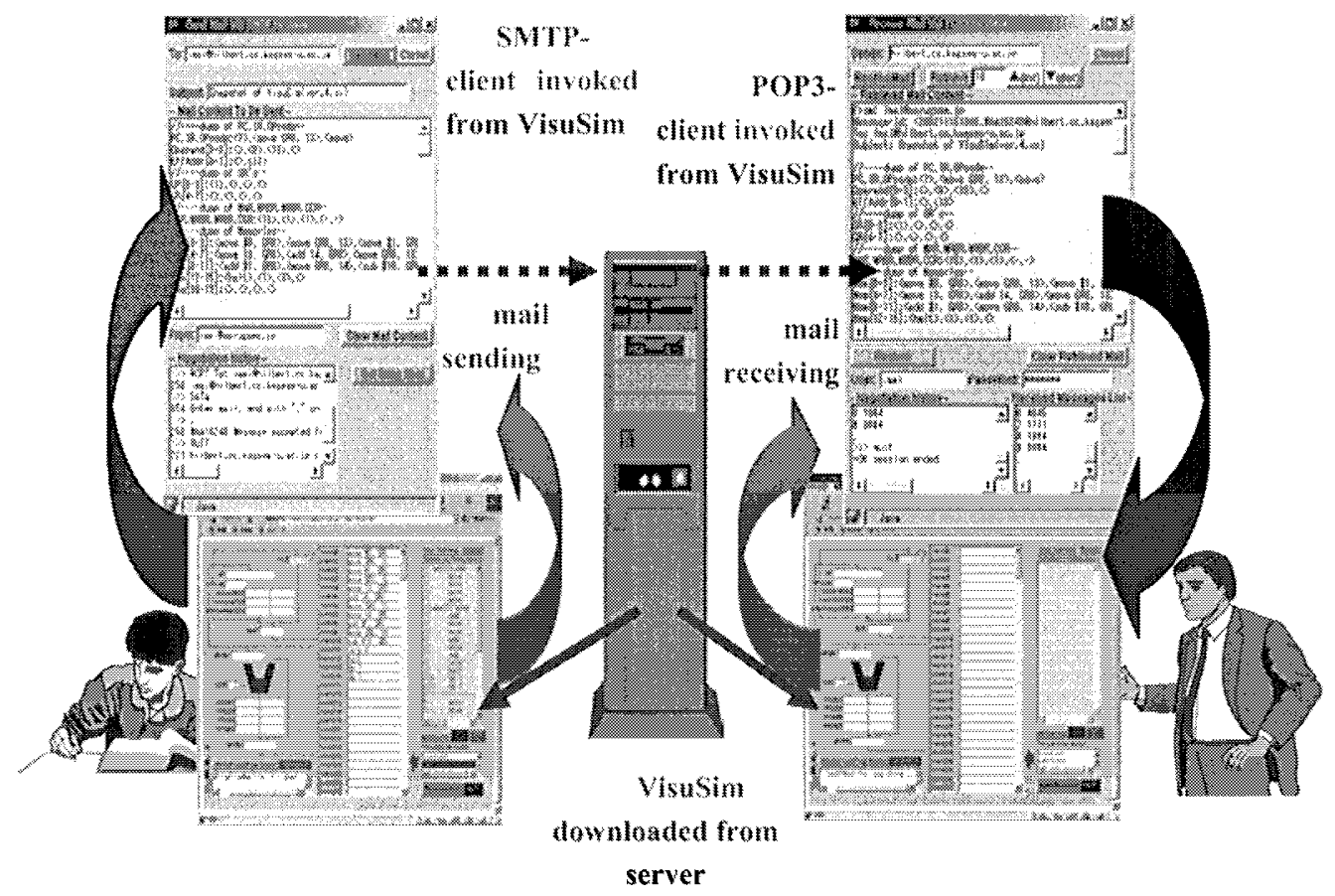

Figure 3. Overview of Collaborative (Distance) Education with VisuSim

(2) Characteristics of POP3-based mail receiver:

As the same functions of SMTP-based mail sender, this mail receiver has the following two characteristics different from others; namely easy restoring data of all the registers/memory in VisuSim from content of the received mail and IP address direct specification of mail server. On the other hand, it has some weaknesses to handle several kinds of mail messages. For example, it cannot process mail with attached files. Although it reads out the specified mail from server, it shall not delete that mail data from the server. So another mailer will be able to process non-deleted mails with attached files. The mail facility of VisuSim may be useful not only for consulting communication between students and teacher but also for on-line submission of students' reports to teacher. Figure 3 also shows such a communication between students and teacher. The mail sender and receiver of VisuSim can handle message in Japanese as well as English. With such embedded mail handler of VisuSim, students who need some kind of advice can send help mail to others and obtain suitable advices from teacher and/or other students 
who know more than them. As a result, almost all students will benefit from submitting their reports to the teacher by means of the above mail service.

\subsection{Function of Simulator}

Structure of program is sometimes discussed in a lecture of computer system and assembly programming exercise. Program including iteration with index registers belongs to a class of difficult subject for beginner of computer. In general, iteration is implemented with combination of conditional jump, indirect addressing and so on. Beginners may suffer from lack of suitable education tools which support illustrating how relatively complicated iteration is processed in a computer. For example, it is useful to explain the concept of effective addressing in order to show the distinction between direct addressing, indirect one and immediate value one graphically. Explanation of mechanism for subroutine call and return is one of the most essential themes in the computer system. It must involve calling sequence with parameter passing and allocation of stack and/or heap. Recursive subroutine call may be frequently included in the above themes. As stack frame is easily handled by a few suitable instructions, recursive programs can be written at assembly programming level. It is very important for students to be given a graphical explanation of the internal behaviour and structure of computer, for example, how stack area grows and reduces.

Assembly programming exercise is available through an effective application of our visual simulator as well as it is used to illustrate the internal behaviour and structure of computer. Therefore, relatively complicated iteration processing can be realized with such addressing and interpreted by VisuSim. It is one of the important characteristics that the detail of programming techniques may be explained, which could be hardly understood with only high-level programming languages, such as parameter passing between subroutines and manipulation of data structure and/or array. It is effective that the detail of programming techniques may be explained, which could be hardly understood with only high-level programming languages.

\section{APPLICATION OF VISUSIM}

This sections shows actual cases of using our visual simulator, VisuSim.

(1) Actual Lecture of Computer System

In the classroom lecture such as Computer System and Information Literacy, utilization of VisuSim can advance students' interest in the internal behavior and structure of computer system. Based on sample programs, each 
student challenges to write assembly program and investigate how it is processed inside a computer. With VisuSim as visual education tool, it is available for beginners to understand visually several mechanisms that many kinds of program are processed in computer. It is more effective to educate how computer works by means of VisuSim than by lecture with abstractive explanations. Figure 4 shows actual lecture of Computer System with display projection of VisuSim onto a large screen.

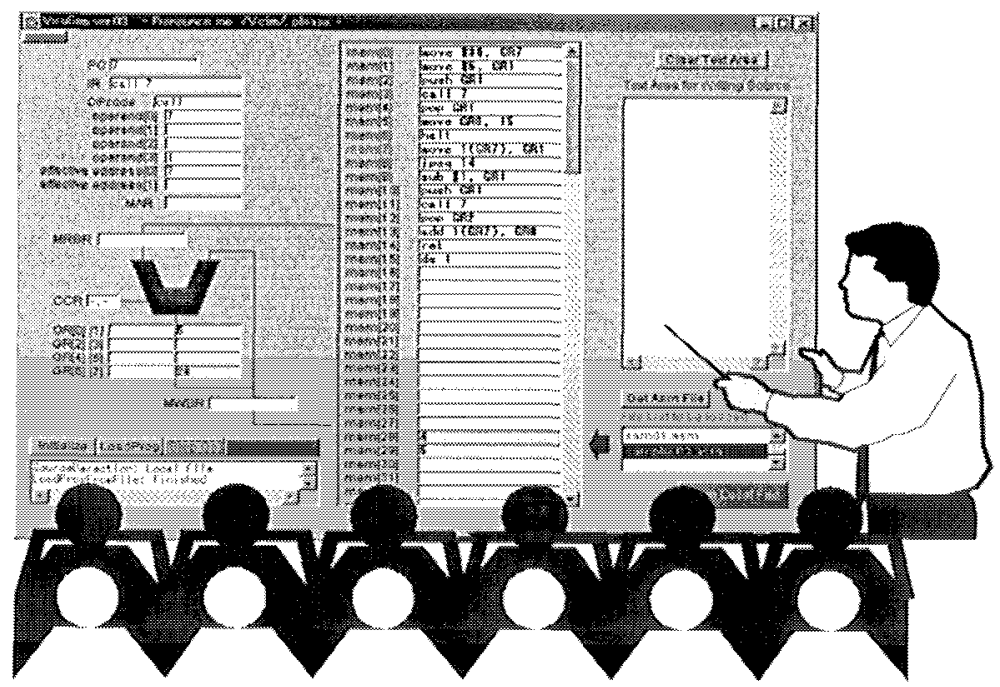

Figure 4. Image of Lecture using VisuSim

(2) Application to Assembly Programming Exercise

Assembly programming capability is essential for education of information systems engineering. This capability can be obtained from not only by lecture on computer system but also by practical exercise. From our Web server, users can obtain other examples of assembly programming such as selection with several conditional jump instructions, repetition with indexed addressing, and so on. Recursive call is one of typical medium-level themes about assembly programming. For example, calculation of factorial and resolution of Hanoi's tower are suitable themes for beginners' learning recursion. Because stack-related instructions are equipped, VisuSim can provide a simple assembly-programming environment to describe recursive subroutine call.

Consequently, combination of two execution modes will provide a powerful debugging facility. In the class of assembly programming exercise, for example, such a debugging facility has been employed to verify whether programs work correctly or not. It is also effective to explain the correspondence between description of high-level language and the 
behaviour of computer such as numbers of repetition and the content of loop control variable in the specific general-purpose register.

\section{CONCLUSION}

The system configuration and some applications of our visual simulator, VisuSim are introduced in the paper. We can conclude our study as follows:

a) By use of VisuSim, a graphical demonstration is available in classroom lecture on Computer System and Assembly Programming, so that it is easy and transparent for even beginners to understand the internal behavior and structure of computer more precisely;

b) Help messages and suitable sample programs can be obtained from a Web server. Embedded mail handler can be provided for communication between its users. It is useful for students to practice with VisuSim not only in the classroom lecture but also after school and/or at home;

c) VisuSim can provide an environment for distant learning for assembly programming. And we may possibly apply VisuSim to an actual example of collaborative learning.

\section{ACKNOWLEDGEMENTS}

The authors are thankful to Prof. Gancho Vachkov, Prof. Hitoshi Inomo, Prof. Wataru Shiraki, and Dean Hiroshi Ishikawa of Kagawa University for their constructive advices. This study is partly supported by grand 12040107 in aid for scientific research from the Ministry of Education, Culture, Sports, Science and Technology.

\section{REFERENCES}

[1] Chung, G.K.W.K., Harrmon, T.C., Baker, E.L. "The impact of a simulation-based learning design project on student learning", IEEE Transaction on Education, 44(4), 390-398 (2001).

[2] Johnson, M., Craig, B. "Computer system pedagogy using logic simulation", Proceedings of International Conference on Computer in Education, Vol. 1. 703-704, 2002

[3] Miura, Y., Kaneko K., Nakagawa M. "Design and Prototyping of an Educational Computer System Simulator ", Proceedings of International Conference on Computer in Education, Vol. 1. 673-674, 2003 